\title{
Global Medicine als Chance für die Schweiz?
}

Gieri Cathomas,

Rainer Arendt

Double Check - Swiss Academic Center for Checkups and Second Opinions, Zürich
Korrespondenz: Gieri Cathomas Double Check Stadelhoferstrasse 22 CH-8001 Zürich

check@doublecheck.ch
Die Gesundheitskosten steigen weiter, bereits setzt die Schweiz 10,6\% ihres Bruttosozialproduktes für die Versorgung ihrer Bevölkerung ein - übertroffen werden wir nur von Frankreich und den USA mit 11\% beziehungsweise 16\% [1]. Neben dem Sparen sind neue Ertragsquellen erforderlich. Im Zentrum steht dabei der Medizintourismus. Immer mehr Patienten aus dem Mittleren Osten und dem ehemaligen Ostblock, wo qualitativ hochstehende Medizin nur bedingt verfügbar und kaum Vertrauen in die lokale Medizin vorhanden ist, suchen ärztlichen Rat in der Schweiz und finanzieren so unser Gesundheitswesen mit.

\section{Was ist gefragt?}

Die ausländischen Patienten suchten weniger Rat in der Primärprävention als vielmehr eine Zweitmeinung bei bestehenden medizinischen Problemen. Dies zeigt eine Analyse bei den ersten 200 Patienten des medizinischen Centers «Double Check» in Zürich. Knapp 95\% der Patienten kamen mit vorbekannten Diagnosen zur Überprüfung der Diagnostik und Therapieentscheidung in die Schweiz. Im Vordergrund stehen HerzKreislauf-Erkrankungen, gastrointestinale Leiden sowie endokrinologische Probleme (Abb. 1). Im Durchschnitt suchten etwas mehr Männer (58\%) nach medizinischen Dienstleistungen in der Schweiz als Frauen. Das Durchschnittsalter betrug knapp über 45 Jahre. Dieses überraschend tiefe Durchschnittsalter zeigt deutlich das höhere Risikoprofil und tiefere Erkrankungsalter bei den Patienten aus den Hauptländern. So wiesen bei Ankunft in der Schweiz die Patienten bereits durchschnittlich 2,5 Diagnosen auf. Abbildung 2 zeigt, dass nur ein sehr geringer Teil der Patienten (aktuell ca. 6\%) ausschliesslich für sogenannte Vorsorgeuntersuchungen in die Schweiz kommt.

\section{Gesundheitstourismus in der Schweiz}

Vor allem Westschweizer Kliniken behandeln heute zahlreiche Patienten aus dem Mittleren Osten, während in der Deutschschweiz das Universitätsspital Basel sowie das an akademische Institutionen angegliederte «Double Check» einen überproportionalen Anteil an ausländischen Patienten haben (Abb. 3.).

Da es sich bei diesen Patienten um Selbstzahler aus dem Ausland handelt, belasten sie die öffentliche Hand nicht. Verlässliche Zahlen zur Marktgrösse des Medizintourismus in der Schweiz wurden noch nicht erhoben. Man geht jedoch von einem Umsatz von gegen 900 Millionen Franken pro Jahr aus.

Um den Schweizer Spitälern eine Marketingplattform zu bieten, haben die Osec und Schweiz Tourismus den Verein Swiss Health gegründet. Ziel des Ver-
La médecine globale, une chance pour la Suisse?

Les coûts de santé ne cessent d'augmenter. Dès à présent, la Suisse consacre $10,6 \%$ de son produit social brut aux soins à la population. Outre l'épargne, il faut trouver de nouvelles sources de revenus. La démarche se focalise sur le tourisme médical. Des patients toujours plus nombreux du Moyen-Orient et de l'ancien bloc de l'Est, pour lesquels une médecine de qualité n'est pas toujours disponible et qui font peu confiance aux praticiens locaux recherchent un soutien médical en Suisse. Plutôt qu'un conseil de prévention primaire, ils sollicitent un deuxième avis sur des problèmes médicaux dont ils sont atteints. Tout juste $\mathbf{9 5} \%$ des patients disposent déjà d'un ou de plusieurs diagnostics et recherchent une opinion supplémentaire dans le système de santé suisse très développé. Les consultations portent principalement sur les maladies cardio-vasculaires, les maux gastrointestinaux et les problèmes endocrinologiques. L'utilité du tourisme médical est évidente: les hôpitaux peuvent admettre des patients étrangers qui paient leurs soins ce qui leur permet de mieux utiliser leurs infrastructures et d'améliorer leurs bilans. De tels revenus supplémentaires bénéficient aux assurés du régime général et peuvent contribuer à assurer un accès aux soins optimal pour tous à moyen terme.

eins ist es, die Schweiz als Medizintourismusdestination zu vermarkten. In der Startphase wird Swiss Health auch mit Bundesgeldern unterstützt [2].

\section{Ausland erfolgreich in Medizintourismus}

Die Schweiz ist in diesem Markt nicht alleine: Amerikanische Spitzenkliniken behandeln seit jeher Patienten aus aller Welt. Mayo Clinic, Cleveland Clinic, Johns Hopkins usw. nutzen ihr Renommee, um so zusätzliches Einkommen zu erwirtschaften. Ähnliches spielt sich in London am Imperial College und an anderen Universitätskliniken des United Kingdom ab unter anderem dank der traditionellen Beziehungen zu früheren Kolonien. Auch deutsche Zentren haben 
sich gerüstet, vor allem München, Hamburg und die Region Köln-Bonn. 2007 besuchten knapp 71000 ausländische Patienten deutsche Kliniken, was einer Zunahme von 11,4\% gegenüber 2006 (ca. $63500 \mathrm{~Pa}$ tienten) entspricht. Ein Grossteil dieser Patienten kommt allerdings aus den Nachbarländern (Holland, Belgien, Frankreich, Polen und Österreich) [3]. Von den asiatischen Ländern sind Thailand mit dem Bumrungrad Hospital in Bangkok sowie der Stadtstaat Singapur nennenswert. Die geographische Aufteilung der Anbieter kann Abbildung 4 entnommen werden.

\section{Stärken und Schwächen der Schweiz}

Die Schweiz steht grundsätzlich gut da: Neben Tradition verfügt unser Land über hohe Glaubwürdigkeit und ausgezeichnetes Ansehen sowie über ein hervorragendes Gesundheitssystem. Auch hat die Schweiz

\section{Abbildung 1}

Anzahl Diagnosen bei den ersten 200 Patienten, aufgeteilt nach Fachgebieten. (Quelle: Double Check - Swiss Academic Center for Checkups and Second Opinions, Zürich)

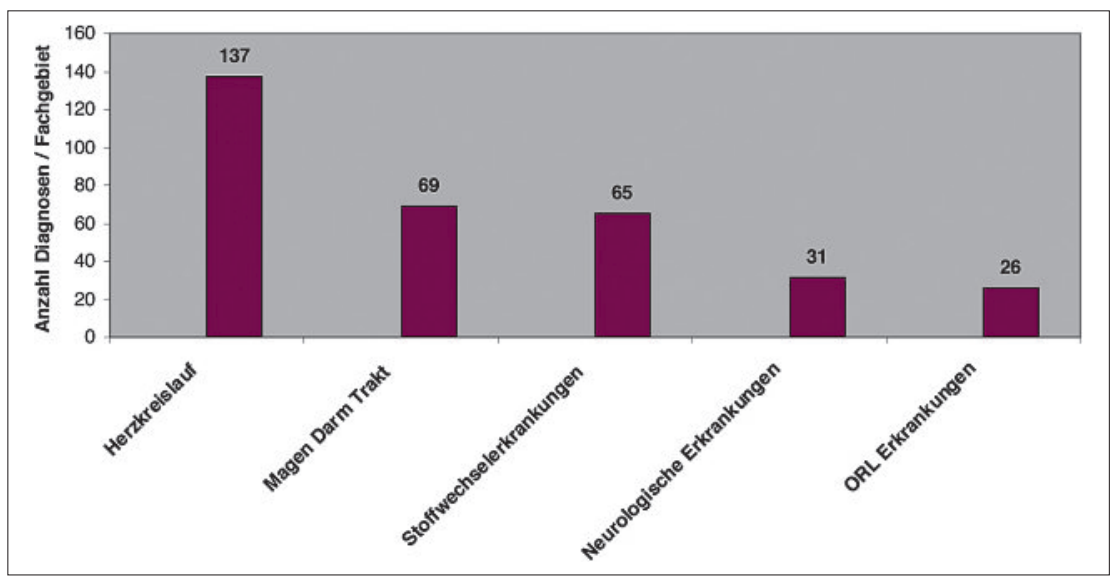

Abbildung 2

Prozentuale Verteilung der Anzahl der Diagnosen pro Patient.

(Quelle: Double Check - Swiss Academic Center for Checkups and Second Opinions, Zurich)

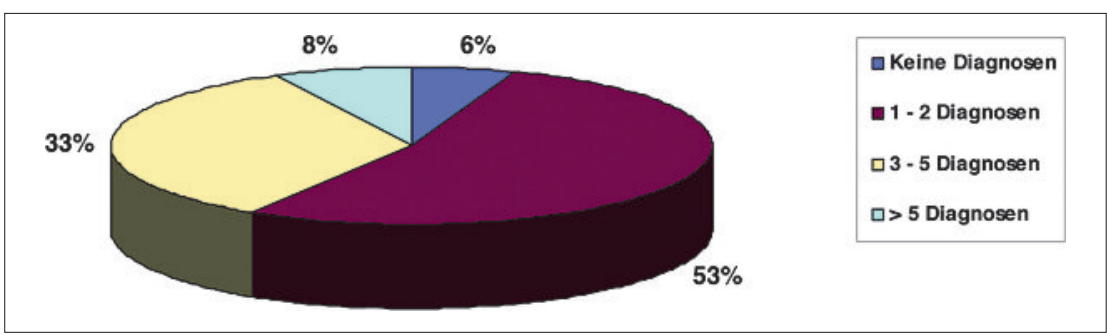

Abbildung 3

Prozentuale Verteilung der Herkunftsregionen der untersuchten 200 Patienten. (Quelle: Double Check - Swiss Academic Center for Checkups and Second Opinions, Zurich )

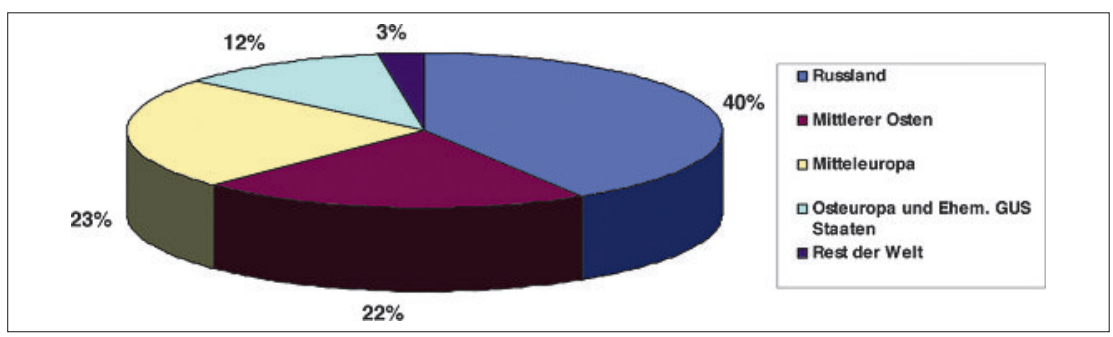

grosse Internisten (Wilhelm Löffler, Ernst R. Froesch, Andreas Grüntzig), Pädiater (Guido Fanconi, Andrea Prader) und Chirurgen (Åke Senning, Mahmut Gazi Yasargil, Maurice E. Müller) hervorgebracht. Unsere Universitätskliniken sind auf vielen Gebieten führend und verfügen über international hochangesehene Mediziner.

Swissness steht weiterhin für Glaubwürdigkeit und Vertrauen - beides Werte, die für Patienten besonders wichtig sind. Die Neutralität hat uns aus Konflikten herausgehalten, was neben dem hohen Lebensstandard und der Qualität unserer Produkte zum Ansehen unseres Landes beitrug. Nicht zuletzt zählt auch die geographische Lage: Die Schweiz liegt inmitten von Europa und doch ausserhalb der überregulierten Welt. Insbesondere Zürich und Genf verfügen mit ihren internationalen Flughäfen und Hotels über die nötige Infrastruktur.

Allerdings ist die Schweiz spät im Rennen. Global Medicine wurde erst kürzlich bei uns wiederentdeckt. Die Konkurrenz hat Erfahrung, Ansehen und etablierte internationale Verbindungen. Die neuen Player aus Südostasien sind sehr flexibel und passen sich ändernden Umständen schnell an, während Service und Kundenorientierung in Schweizer Spitälern bis vor kurzem nicht grossgeschrieben wurden. Aktuelle Entwicklungen bieten aber zusätzliche Chancen für die Schweiz. Beispielsweise ist es sehr beschwerlich geworden, in die USA einzureisen. Die Mayo Clinic wies aufgrund des Rückgangs ausländischer Patienten und der Finanzkrise 2008 ihr schlechtestes Ergebnis aus.

\section{Ethische und rechtliche Aspekte}

Selbstverständlich sind bei der Beratung oder allenfalls notwendigen Behandlungen ausländischer Patienten ethische und rechtliche Standards einzuhalten. Dazu gehört die Einhaltung der Prinzipien der evidenzbasierten Medizin, denn in diesem Markt besteht die Gefahr von Überdiagnostik bzw. Übertherapie $[4,5,6,7,8,9,10,11,12]$. Entsprechend ist eine permanente Qualitätskontrolle unabdingbar, beispielsweise mittels Publikation der Ergebnisse. Weiter müssen öffentliche Institutionen durch entsprechende Massnahmen sicherstellen, dass die Verfügbarkeit diagnostischer und therapeutischer Angebote für die einheimischen Patienten nicht eingeschränkt wird. Schliesslich muss für die ausländischen Patienten ein ausreichender Versicherungsschutz gewährleistet sein.

\section{Nutzen für Schweizer Gesundheitswesen}

Der Nutzen von Medizintourismus ist klar: Spitäler können mit geringen Investitionen selbstzahlende ausländische Patienten aufnehmen, dadurch ihre Auslastung erhöhen, Stellen schaffen und ihre Bilanzen verbessern. Solche zusätzlichen Erträge kommen auch Allgemeinversicherten zugute und können mittelfristig helfen, eine optimale Versorgung aller sicherzustellen. Es wird nicht eine Zweiklassenmedizin gefördert, zumal gerade in den Ballungsräumen 


\section{Abbildung 4}

Geographische Verteilung nach den häufigsten Herkunftsländern der Patienten (grün) sowie eine Auswahl der wichtigsten Anbieterländer von medizinischen Dienstleistungen weltweit (rot).

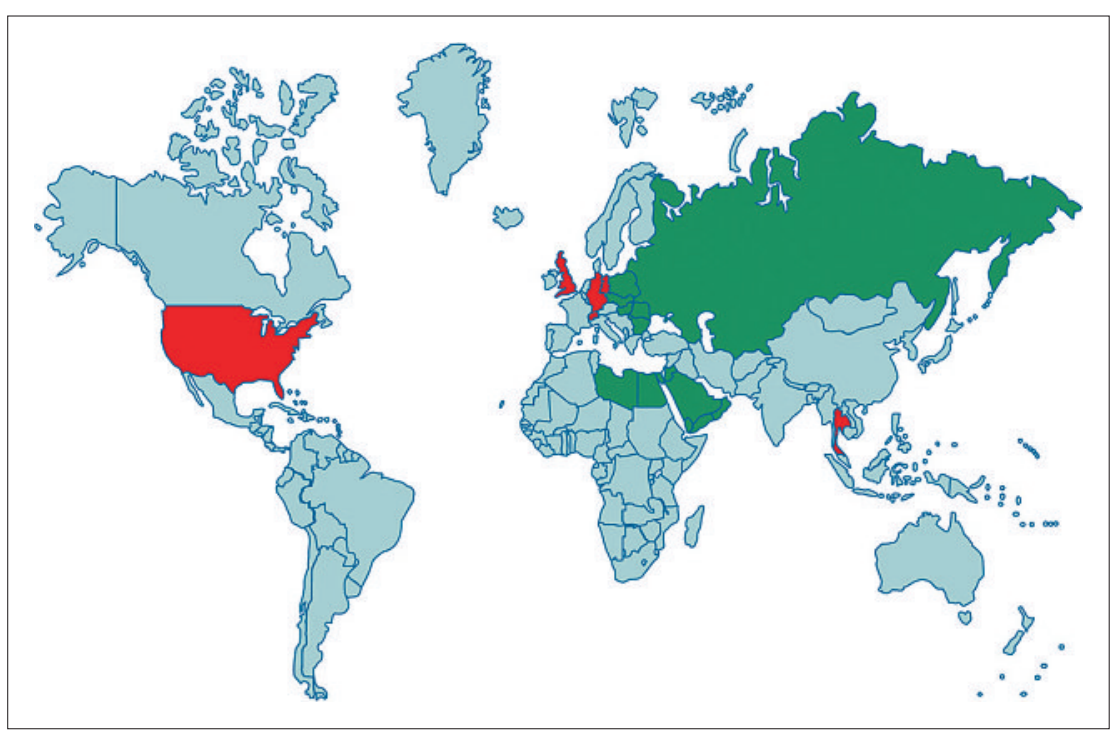

eine medizinische Überversorgung besteht. Mit rund 350 Akutspitälern bei 7,5 Mio. Einwohnern weist die Schweiz weltweit eine der höchsten Spitaldichten auf. Aktuell bieten in unserem Land 26 kardiologische Zentren medizinische Dienstleistungen an, was im Vergleich zu Dänemark oder den Niederlanden einer fast fünf Mal höheren Dichte entspricht.

\section{Wie weiter?}

Die Schlüsselfaktoren für Erfolg im internationalen Medizintourismus heissen individueller Service, Kompetenz und Qualität - letztere sind im Schweizerischen Gesundheitswesen vorhanden. Gerade in Zeiten stark steigender Krankenkassenprämien und der nach wie vor unsicheren Wirtschaftslage sollten Ertragsoptimierungen durch ausländische Patienten im Gesundheitswesen genutzt werden. Akademische Zentren mit Tradition und Ausstrahlung haben medizinisch die Nase vorn, sie sollten ihren Service jedoch dem Niveau der etablierten Privatkliniken anpassen. Gute Voraussetzungen für einen erfolgreichen Markteintritt bietet beispielsweise ein Public-Private-Partnership mit akademischen Institutionen, an denen am meisten Knowhow vorhanden ist. In der Tat können damit vor allem der Erstkontakt und der individuelle Service für ausländische Patienten rasch den internationalen Standards angepasst werden. Deutschland und auch die USA haben sich bereits erfolgreich auf dem internationalen Patiententourismusmarkt etabliert - die Zeit drängt.

\section{Literatur}

1 OECD Health Data; Datenstand Juli 2009 und Bundesamt für Statistik, Stand 2007.

2 Medienmitteilung OSEC vom 27. November 2008: OSEC und Schweiz Tourismus gründen Verein zur Vermarktung der Gesundheitsdestination Schweiz.

3 Juszczak J. Ergebnisse der 4. Marktstudie von internationalen Patienten in deutschen Kliniken. Präsentation in Bonn am 16. September 2009.

4 European Society of Hypertension European Society of Cardiology Guidelines for the management of hypertension. J. Hypertension. 2003; 21:1011.

5 Prognostic value of multiple slice computed tomography and gated SPECT in patients with suspected coronary artery disease. J. Amer. Coll. Cardiol. 2009;53:623.

6 Cardiac computed tomography: indications, applications, limitations, and training requirements. Report of the writing group by Schroeder et al. Eur. Heart J. 2008;29:531.

7 Steffel J, Lüscher TF. Predicting the development of atherosclerosis. Circulation. 2009;119:919.

8 European Society of Cardiology Textbook of Cardiovascular Medicine. Eds. Camm JA, Lüscher TF. Serruys PW. Oxford: Blackwell; 2005.

9 Lüscher TF (Hrsg.). CARDIX - Praxismanual der kardiovaskulären Medizin. Zürich; 2008.

10 Winawer S, Classen M, Lambert MR, Fried M et al. Colorectal cancer screening. World Gastroenterology Organisation, Guidelines and Publications Committee (Chair: M. Fried). 2007; www.worldgastroenterology.org/colorectal-cancerscreening.html.

11 Herzog et al. Accuracy of low dose computed tomography coronary angiography using prospective ECG-triggering. Eur Heart J. 2008;29:3037.

12 Guide to Clinical Preventive Services; 2009. Recommendations of the U.S. Preventive Services Task Force. 